\title{
ANÁLISE DA IMAGEM DIGITAL PARA ESTIMATIVA DA ÁREA FOLIAR EM PLANTAS DE LARANJA "PÊRA"
}

\author{
LEANDRO JOSÉ GRAVA DE GODOY²; RODRIGO SEIGI YANAGIWARA ${ }^{3}$, ROBERTO LYRA VILLAS BÔAS ${ }^{4}$, \\ CLARICE BACKES 5 , CLAUDINEI PAULO DE LIMA ${ }^{6}$
}

RESUMO - Objetivou-se, com o trabalho, avaliar dois métodos de estimativa da área foliar, em plantas de laranja "Pêra", pela análise da imagem digital obtida com scanner e câmera fotográfica digital. Para determinar a área das folhas, um grupo de discos foi colocado sobre um leitor de scanner, sendo que a imagem obtida foi armazenada. Os mesmos grupos de discos foram fixados sobre cartolina branca e fotografados com câmera fotográfica digital. As imagens obtidas da câmera fotográfica e do scanner foram processadas utilizando ferramentas de um editor de imagem que permite a contagem de pixels de determinada cor, no caso verde. Para a comparação dos métodos, os discos foram submetidos a integrador óptico de área foliar modelo LICOR-3100, utilizando os mesmos agrupamentos. Foram coletadas 20 folhas (cinco em cada quadrante da planta) por parcela de um experimento para comparação de fertilizantes comerciais e doses de zinco, aplicados via foliar, em plantas de sete anos de idade. O experimento foi composto de sete tratamentos e quatro repetições, num total de 28 parcelas. Os dois métodos apresentaram alta correlação com a área estimada pelo integrador óptico de área, considerado como método de referência. O método da análise da imagem obtida com câmera fotográfica, na resolução de 5.0 megapixel, foi mais precisa quando comparada à área estimada pelo integrador óptico de área.

Termos para indexação: Citrus sinensis L., câmera fotográfica, scanner e folhas.

\section{DIGITAL IMAGE ANALYSIS FOR ESTIMATIVES OF LEAF AREA IN “PÊRA" ORANGE PLANTS}

ABSTRACT - This work had as purpose to evaluate two methods of the estimative of leaf area, in "Pêra" orange plants, by the digital image analysis obtained with scanner and digital camera. To determine the leaf area, one group of discs was placed on a reading scanner and the image obtained was stored. The same groups of discs were fixed over a white paper and photographed with a digital camera. The images obtained from the digital camera and the scanner was processed utilizing an image editor's tool that allows the pixels counting of a specific color, in this case green. To compare these two methods the discs were submitted to an optic integrator of green leaf, LICOR-3100 model, utilizing the same groups. It was collected 20 leaves (five in each square of the plant) for each parcel of one experiment to compare commercial fertilizers and $\mathrm{Zn}$ doses, applied by leaves spraying, in seven years old "Pêra" orange plants. The experiment comprehended seven treatments and four replications with a total of 28 parcels. The two methods presented high correlation with the estimated area considering the optic integrator of area the reference method. The analysis of the image obtained from the photographic camera, with 5.0 megapixel of resolution, had more precision when compared with the estimated area by the optic integrator.

Index terms: Citrus sinensis L., photographic camera, scanner, and leaves.

\section{INTRODUÇÃO}

A análise de crescimento, segundo Pereira \& Machado (1987), representa a referência inicial da análise da produção das espécies vegetais, requerendo como informações a quantidade de material produzido pela planta (fitomassa) e o tamanho do aparelho fotossintetizante (área foliar), obtidas em intervalos regulares durante o desenvolvimento fenológico da planta. $\mathrm{O}$ conhecimento da área foliar é de grande utilidade para a avaliação de técnicas culturais, como poda, adubação, densidade de plantio e aplicação de defensivos (Tavares-Júnior et al., 2002).

A medida da área foliar está relacionada à determinação ou estimativa da superfície fotossinteticamente ativa. Segundo Benincasa (1988), há diferentes métodos, destrutivos ou não, para se estimar a área foliar, a maioria com alto grau de precisão. Os métodos utilizados para a medição da área foliar empregam medidores eletrônicos e técnicas de planimetria (Kvet \& Marshall, 1971). O uso da planimetria torna-se difícil em folhas com bordas irregulares (Gonçalves et al., 2002), além de ser bastante trabalhoso e demorado. Os integradores ópticos de área foliar (ex.: LI-COR

'(Trabalho 006-07). Recebido em: 03-01-2007. Aceito para publicação em: 19-04-2007.

${ }^{2}$ Prof. Dr., UNESP - Câmpus Experimental de Registro, Rua Tamekishi Takano, 5, 11900-000, Registro-SP; legodoy@registro.unesp.br.

${ }_{3}^{3}$ Aluno do Departamento de Recursos Naturais/ Ciência do Solo, Faculdade de Ciências Agronômicas - UNESP, C.P. 237, 18.610-907, Botucatu-SP. rsyanagivara@fca.unesp.br.

${ }^{4}$ Prof. Dr. Do Departamento de Recursos Naturais/Ciência do Solo, Faculdade de Ciências Agronômicas - UNESP, C.P. 237, 18.610-907, Botucatu-SP. rlvboas@fca.unesp.br.

${ }_{5}^{5}$ Doutoranda do Departamento de Produção Vegetal/Horticultura, Faculdade de Ciências Agronômicas - UNESP, C.P. 237, 18.610-907, Botucatu-SP. cbackes@fca.unesp.br, Bolsista CAPES.

${ }^{6}$ Doutorando do Departamento de Produção Vegetal/Agricultura, Faculdade de Ciências Agronômicas - UNESP, C.P. 237, 18.610-907, Botucatu-SP. cplima@fca.unesp.br, Bolsista CNPq 
3100, Area meter) são aparelhos bastante precisos, mas não são fabricados no Brasil e, por isso mesmo, são muito caros e de difícil manutenção. Além disso, o equipamento tem dimensões que limitam a leitura em folhas muito largas. Também a nervura central pode ser fator limitante para o uso desse equipamento, principalmente quando essas são de maior diâmetro.

Outro método é a estimativa da área foliar realizada por meio de fatores de correção calculados de medições simples e não-destrutivas, como através do produto do comprimento ao longo da nervura principal e a largura máxima (Barros et al., 1973; Bianco et al., 2003) e comprimento da folha (Pereira, 1987).

A utilização de imagens digitais obtidas por scanner e processadas por programas específicos foi realizada por TavaresJúnior et al. (2002), que utilizaram o SIARCS 3.0 (Sistema Integrado para Análises de Raízes e Cobertura do Solo) desenvolvido pela EMBRAPA/ CNPDIA. Neste método, para estimar a área foliar de cafeeiro em relação aos métodos do retângulo circunscrito aos limbos foliares e dos discos foliares, ele apresentou maior exatidão.

Atualmente, a obtenção de imagens digitais com boa resolução pode ser realizada através de câmeras digitais de modo rápido e simples. Richardson et al. (2001) obtiveram alta correlação $\left(r^{2}=0,99\right)$ entre a taxa de cobertura do solo pela grama pela análise da imagem de câmera digital de 1.280 x 960 pixels (pixel é a menor unidade que compõe uma imagem digital, e que contém os atributos de cor de cada ponto) e a taxa de cobertura calculada.

Uma das dificuldades dos métodos que utilizam as imagens digitais para estimar a área foliar é a necessidade de um programa computacional capaz de processar a imagem, calculando a área desejada, pois estes geralmente são de custo elevado. Godoy (2005) encontrou resultados precisos utilizando dois programas comumente utilizados: um, o editor de imagem (Corel Photo Paint ${ }^{\mathrm{TM}}$ ) e outro, uma planilha de cálculo (Microsoft Excel 2000 ${ }^{\mathrm{TM}}$ ) para calcular a taxa de cobertura do solo com base em imagens digitais obtidas de câmera fotográfica.

Objetivou-se, com o presente trabalho, avaliar a estimativa da área foliar pela análise da imagem digital obtida com scanner e câmera fotográfica digital em editor de imagem (Corel Photo Paint ${ }^{\mathrm{TM}}$ ), assumindo como referência os resultados obtidos com o integrador óptico de área foliar (LI-COR 3100).

\section{MATERIAL E MÉTODOS}

Para a calibração inicial dos métodos para a determinação da área foliar, foram confeccionados 16 discos de papel-cartão da cor "verde-floresta" (padrão RGB - Red $=34$; Green $=139$ e Blue $=$ 34), simulando a folha de uma planta, com diâmetro de $5,5 \mathrm{~cm}$. Para cada método, foram utilizados grupos de $1 ; 2 ; 4 ; 6 ; 8 ; 10$ e 12 discos correspondendo a 23,$76 ; 47,52 ; 95,03 ; 142,55 ; 190,07 ; 237,58$ e $285,10 \mathrm{~cm}^{2}$. Cada grupo foi distribuído sobre um leitor de scanner (HP2400) e a imagem obtida em uma resolução de 300 dpi ("Dots Per Inch" - pontos por polegada) sendo armazenada, em um microcomputador, no formato JPEG ("joint photographic experts group", .jpg) correspondente a 3.508 x 2.480 pixels. Os mesmos grupos de discos foram colocados sobre uma cartolina branca, em uma mesa, com a câmera fotográfica digital, fixa em suporte em forma de "L" invertido, posicionada paralelamente à cartolina, numa altura de $30 \mathrm{~cm}$, de modo que todos os discos, quando no maior agrupamento (12), estivessem dentro da área de captação da imagem da câmera (Figura 1). Para evitar um "encurvamento" dos discos (o que poderia ocorrer com folhas de plantas também), que poderia reduzir a área amostrada, foi utilizado um vidro totalmente transparente colocado sobre os discos, mantendo-os prensados como no scanner com a tampa fechada. Foram obtidas imagens na resolução de 3.1 e 5.0 "megapixel", correspondente a $2048 \times 1.536$ (72 dpi) e $2.592 \times 1.944$ pixels (72 dpi), respectivamente, sem a utilização de flash para evitar o reflexo do brilho deste no vidro sobre os discos. Para garantir a iluminação, as imagens foram obtidas em sala com boa iluminação, utilizando lâmpadas fluorescentes. Após a obtenção das imagens com a câmera digital, estas foram descarregadas em um microcomputador para posteriormente serem processadas.

As imagens obtidas da câmera fotográfica e do scanner foram processadas utilizando ferramentas de um editor de imagem (Corel Photo Paint) que permite a contagem de pixels de determinada cor, no caso, verde.

Para a comparação dos métodos, os discos de papel-cartão foram submetidos a um integrador óptico de área foliar (LICOR3100) do Departamento de Botânica do Instituto de Biociências da UNESP de Botucatu, utilizando os mesmos agrupamentos. Os resultados foram anotados e depois copilados por microcomputador.

Para a validação dos métodos, utilizou-se de folhas de laranjeiras "Pêra" enxertadas em limoeiro "Cravo", com sete anos de idade, no espaçamento de 7 x 4 m, localizadas na Fazenda Três Irmãos, em Pratânia-SP. Essas plantas fazem parte de um experimento que estuda efeitos de adubação que compara fertilizantes comerciais e doses de zinco, aplicado via foliar. Foram coletadas 20 folhas (cinco em cada quadrante da planta) por parcela do experimento, composto de sete tratamentos e quatro repetições, num total de 28 parcelas. Foi escolhido este experimento devido à observação visual da diferença na área foliar entre os tratamentos. Essa variação na área foliar foi considerada favorável para o estudo pretendido com o objetivo de validar os métodos de determinação testados.

Os mesmos procedimentos utilizados para a determinação da área dos discos de papel- cartão foram utilizados para determinar a área das folhas. No método com a utilização do scanner, foram obtidas duas imagens por parcela, pois as 20 folhas não se enquadravam na área de leitura do scanner, sendo divididas em dois grupos de 10 folhas.

Para a padronização nos dois métodos, sempre foi avaliado um quadrado de papel-cartão "verde- floresta" (padrão RGB Red $=34 ;$ Green $=139$ e Blue $=34)$ de $100 \mathrm{~cm}^{2}$.

Os resultados foram submetidos à análise de regressão linear, utilizando programa Sisvar versão 4.6 (Ferreira, 2003).

\section{RESULTADOS E DISCUSSÃO}

No estudo de calibração, todos os métodos apresentaram alta correlação $\left(r^{2}=0,999\right)$ entre a área calculada e a estimada (Figura 2). A estimativa da área pela análise da imagem digital obtida do scanner de mesa, utilizando o Corel Photo Paint, foi 
bastante precisa com coeficiente angular de 1,001 e o intercepto muito próximo de zero $(0,178)$. A utilização do integrador óptico de área (area meter) também permitiu uma excelente estimativa da área com o intercepto muito próximo de zero $(0,122)$; no entanto, o coeficiente angular foi menor do que um $(0,9895)$, significando que este método pode subestimar a área. O ajuste da área estimada pela imagem obtida de câmera fotográfica digital, embora tenha permitido alta correlação entre a área calculada e a estimada, apresentou um intercepto maior que o ajuste obtido pelo integrador óptico e pelo scanner, além de subestimar a área (coeficiente angular de 0,96 e 0,97 para as imagens de 3.1 e 5.0 megapixel, respectivamente).

$\mathrm{Na}$ estimativa da área das folhas de citros, ambos os métodos apresentaram alta correlação com a área estimada pelo integrador óptico de área, considerado como método de referência. A análise da imagem obtida da câmera fotográfica, na resolução de 5.0 megapixel, foi mais precisa (maior coeficiente de determinação) e subestimou menos a área foliar (Figura 4), além de apresentar menor dispersão de erros (Figura 5) que a análise da imagem obtida do scanner (Figuras 3 e 6 ) quando comparada à área estimada pelo integrador óptico de área.
Segundo Tavares-Júnior et al. (2002), a adoção do método que utiliza a imagem digital obtida de scanner e um software (SIARCS versão 3.0), apresenta vantagem econômica devido ao menor custo de obtenção e operacional, comparado ao custo de aquisição e manutenção do integrador óptico de área (LICOR modelo 3100). A utilização de um editor de imagem (Corel Photo Paint), geralmente presente nos computadores de universidades e instituições de pesquisas, pode tornar o método utilizado no presente trabalho mais vantajoso.

As vantagens da utilização da câmera fotográfica na obtenção da imagem das folhas a ser determinada a área, em relação ao scanner, é que esta pode ser obtida sem a necessidade de um computador, podendo ser executada em qualquer local com boa iluminação. Além disso, pode possibilitar a obtenção de uma área amostrada maior do que o scanner, aumentando a altura da câmera fotográfica em relação às folhas (Figura 1). Godoy (2005), trabalhando com a câmera digital, posicionada a $1,6 \mathrm{~m}$ de altura, em relação às folhas de grama esmeralda, conseguiu obter a imagem digital equivalente à área de $2 \mathrm{~m}^{2}$. Esse aspecto facilita a leitura da área foliar em plantas que apresentam grande número de folhas ou folhas muito grandes.

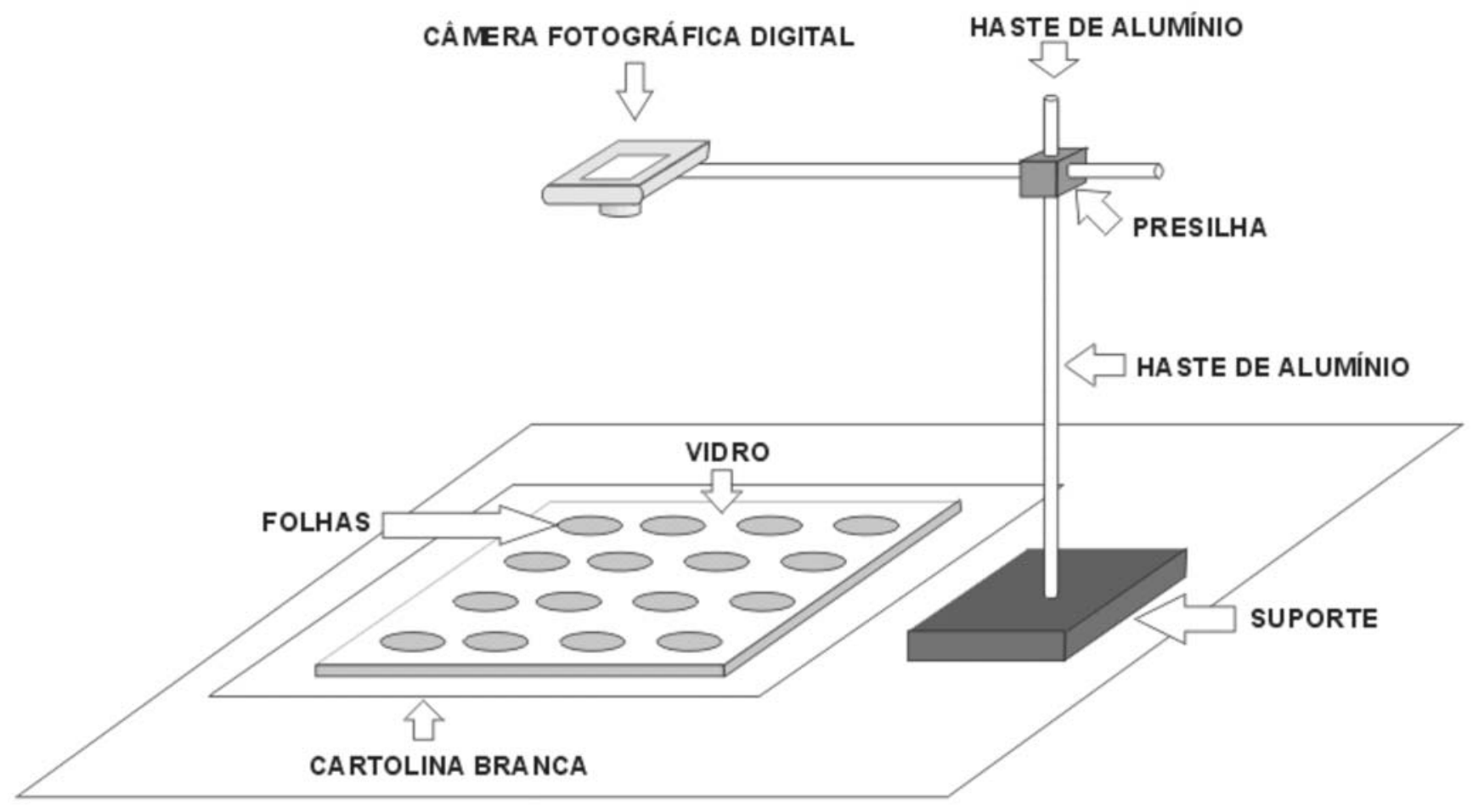

FIGURA 1. Esquema do dispositivo usado na obtenção das imagens com a câmera digital para a determinação da área foliar. 


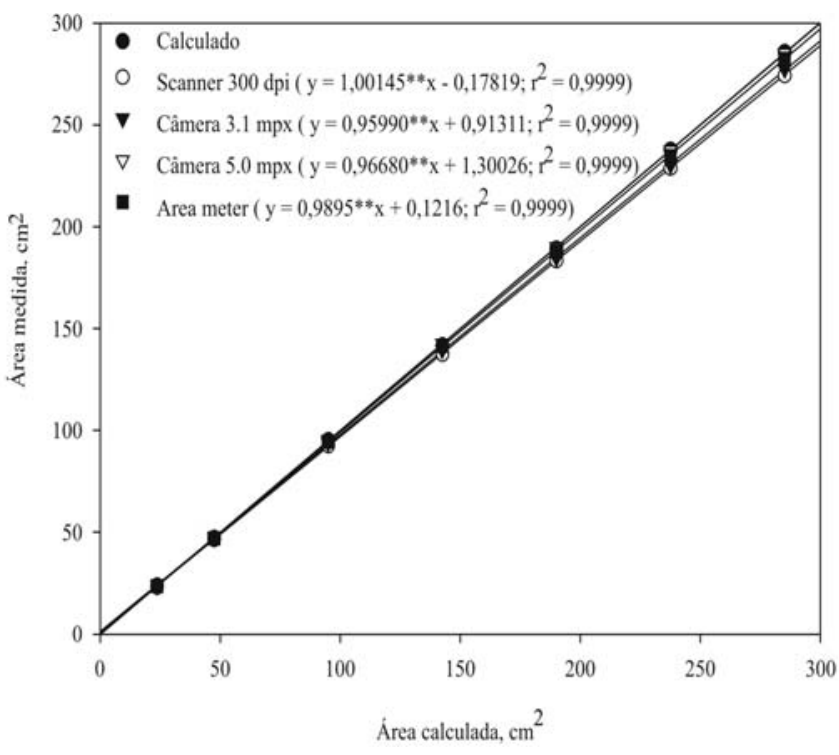

FIGURA 2 - Análise de regressão da área estimada pelo integrador de imagem óptico (Area meter) e pela imagem digital obtida de scanner (300dpi) e câmera fotográfica (3.1 e $5.0 \mathrm{mpx}$ - megapixel).

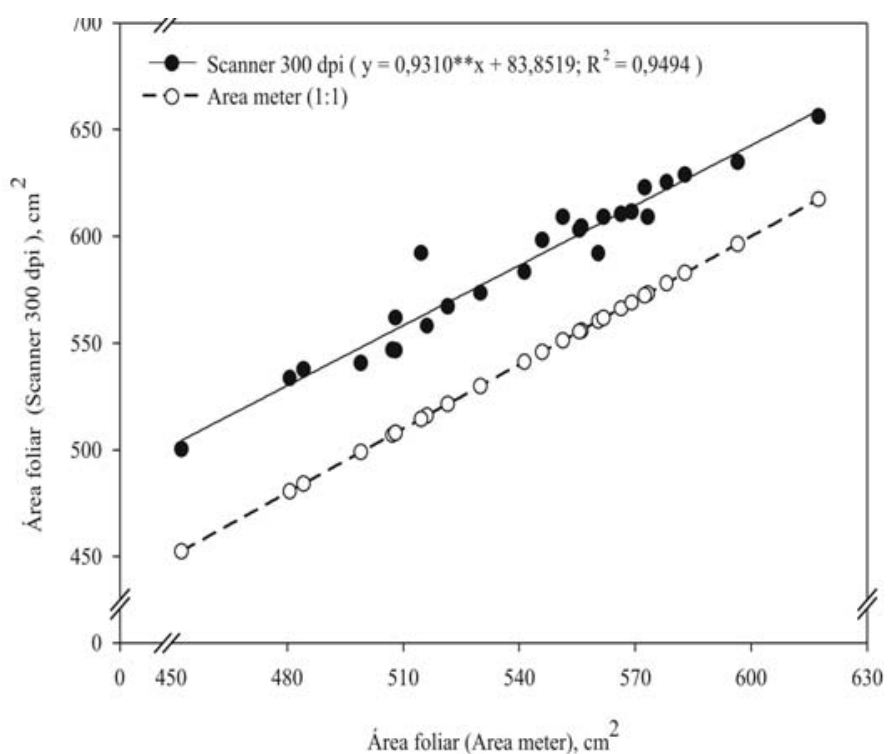

FIGURA3 - Análise de regressão da área estimada pelo integrador de imagem óptico (Area meter) e pela imagem digital de folhas de laranjeira "Pêra" obtida com scanner (300dpi). Cada ponto no gráfico representa $\mathrm{a}$ área total de 25 folhas.

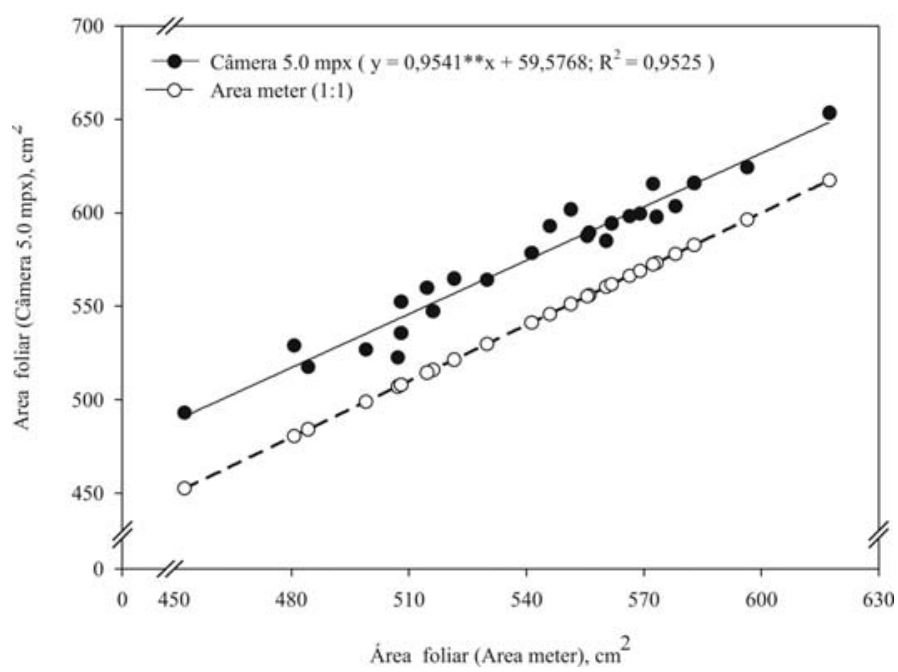

FIGURA 4 - Análise de regressão da área estimada pelo integrador de imagem óptico (Area meter) e pela imagem digital de folhas de laranjeira "Pêra" obtida com câmera fotográfica digital $(5.0 \mathrm{mpx})$. Cada ponto no gráfico representa a área total de 25 folhas.

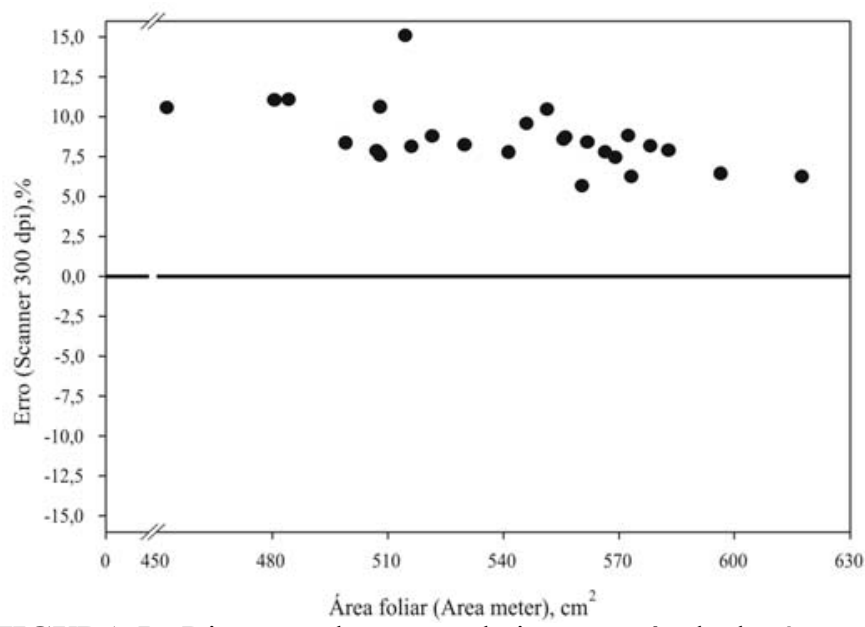

FIGURA 5 - Dispersão dos erros relativos ao método da câmera fotográfica digital

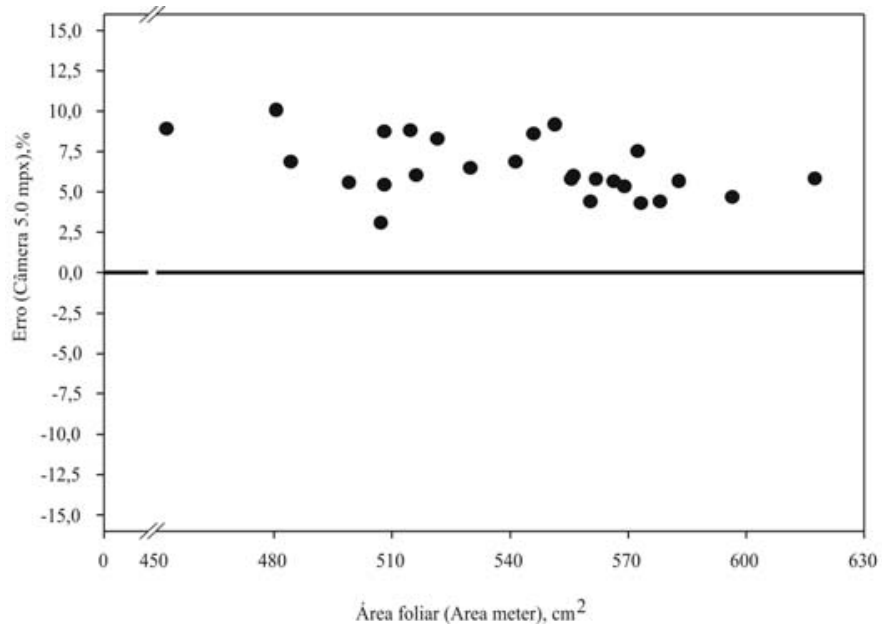

FIGURA 6. Dispersão dos erros relativos aos métodos do scanner. 


\section{CONCLUSÕES}

1 -Os dois métodos apresentaram alta correlação com a área estimada pelo integrador óptico de área, considerado como método de referência.

2-A análise da imagem obtida com câmera fotográfica, na resolução de 5.0 megapixel, foi mais precisa quando comparada à área estimada pelo integrador óptico de área.

\section{REFERÊNCIAS}

BARROS, R.S.; MAESTRI, M.; VIEIRA, M.; BRAGA-FILHO, L.J. Determinação da área de folhas do café (Coffea arabica L. cv. "Bourbon Amarelo"). Revista Ceres, Viçosa, v.20, n.107, p.44-52, 1973.

BENINCASA, M.M.P. Análise de crescimento de plantas: noções básicas. Jaboticabal: UNESP - Câmpus de Jaboticabal, 1988. 41p. BIANCO, S.; PITELLI, R.A.; PITELLI, A.M.C.M. Estimativa da área foliar de Typha latifolia usando dimensões lineares do limbo foliar. Planta Daninha, Viçosa, v.21, n.2, p.257-261, 2003.

GODOY,L.J.G. Adubação nitrogenada para produção de tapetes de grama Santo Agostinho e Esmeralda. 2005. 106f. Tese (Doutorado em Agronomia/Agricultura) - Faculdade de Ciências Agronômicas, Universidade Estadual Paulista, Botucatu, 2005.

GONÇALVES, C.A.A.; CHALFUN, N.N.J.; REGINA, M.A.; ALVARENGA, A.A.; SOUZA, M.T.; ABRAHÃO, E. Estimativa de área foliar da videira (Vitis labrusca L. cv. Folha de Figo) sobre diferentes porta-enxertos. Ciência e Agrotecnologia, Lavras, v.26, n.3, p.500-504, 2002.
FERREIRA, D.F. Sisvar versão 4.6. Lavras: DEX/UFLA, 2004. $32 p$.

HUERTA, S.A. Comparación de métodos de laboratorio y de campo para medir el área del cafeto. Cenicafé, Chinchina, v.13, n.1, p.33$42,1962$.

KVET, J. MARSHALL, J.K. Assessment of leaf area and other assimilating plant surfaces. In: CATZK, J.; JARVIS, P.G. (Eds.). Plant photosynthetic production: manual of methods. The Hague: Junk, 1971.p.517-575.

PEREIRA, A.R. Estimativa da área foliar em milharal. Bragantia, Campinas, v.46, n.1, p.147-150, 1987.

PEREIRA, A.R.; MACHADO, E.C. Análise quantitativa do crescimento de comunidades vegetais. Campinas: IAC, 1987.33p. (Boetim Técnico, 114).

RICHARDSON, M.D.; KARCHER, D.E.; PURCELL, L.C. Quantifying turfgrass cover using digital image analysis. Crop Science, Madison, v.41, p.1.884-1.888, 2001.

TAVARES-JUNIOR, J.E.; FAVARIN, J.L.; DOURADO-NETO, D.; MAIA, A.H.N.; FAZUOLI, L.C.; BERNARDES, M.S. Análise comparativa de métodos de estimativa de área foliar em cafeeiro. Bragantia, Campinas, v.61, n.2, 199-203, 2002. 